Quim. Nova, Vol. 36, No. 2, 230-236, 2013

\title{
DiSTRIBUIÇão DE Zn, Pb, Ni, Cu, Mn E Fe NAS FRAÇõeS do SEDIMENTO SUPERFICIAL dO RIO CACHOEIRA NA REGIÃO SUL DA BAHIA, BRASIL
}

\author{
José Soares dos Santos*, Flávio Mendes de Souza e Maria Lúcia Pires dos Santos \\ Departamento de Ciências Naturais, Universidade Estadual do Sudoeste da Bahia, Estrada do Bem Querer, km 4, 45083-900 \\ Vitória da Conquista - BA, Brasil
}

Recebido em 31/3/12; aceito em 17/8/12; publicado na web em 23/1/13

\begin{abstract}
DISTRIBUTION OF Zn, Pb, Ni, Cu, Mn AND Fe IN SUPERFICIAL SEDIMENT FRACTIONS OF THE CACHOEIRA RIVER IN THE SOUTH OF BAHIA, BRAZIL. The metal distribution in the surface sediment fractions of the Cachoeira River was evaluated based on the fractionation method using a five-step sequential extraction. The determination of metals was made by flame atomic absorption spectrophotometry (F AAS). $\mathrm{Zn}, \mathrm{Pb}$ and $\mathrm{Cu}$ exhibit higher concentrations in the residual fraction of the sediment from sites that receive discharges from urban and industrial zones. High levels of Ni $\left(60 \pm 1\right.$ to $\left.447 \pm 9 \mu \mathrm{g} \mathrm{L}^{-1}\right)$ were found in the river water, which may be detrimental to the "health" of rural communities that utilize the river water for domestic purposes without treatment.
\end{abstract}

Keywords: toxic element; availability of metals; sediment samples.

\section{INTRODUÇÃO}

O uso e a ocupação do solo é um dos fatores mais importantes no controle de qualidade das águas interiores. O Rio Cachoeira localizado na região sul da Bahia, apresenta uma extensão de $50 \mathrm{~km}$ em seu curso, cuja área de drenagem é de $4.830 \mathrm{~km}^{2}$, onde residem aproximadamente 600.000 habitantes. Durante as últimas décadas, vem ocorrendo nesta região uma mudança nos padrões de urbanização devido ao rápido crescimento da população e incremento das atividades industriais, agrícolas e turísticas.

Do ponto de vista antropogênico, a Bacia do Rio Cachoeira é considerada um absorvedouro de dejetos de origens domésticas, industriais e agrícolas. As populações ribeirinhas dependem direta ou indiretamente das águas do Rio Cachoeira ou de seus afluentes. No percurso que corta o perímetro da cidade de Itabuna é possível encontrar indústrias de diferentes setores, entre elas pode-se destacar um empreendimento agroindustrial que descarrega seus resíduos em um tributário do Rio Cachoeira. Por se tratar de uma produção de suínos em larga escala, tal resíduo pode ser rico em nutrientes minerais como, por exemplo, $\mathrm{Zn}$ e $\mathrm{Cu} .^{1-3}$

Além das fontes pontuais, existem ainda os deflúvios superficiais urbanos e agrícolas, os quais consistem em fontes difusas. O deflúvio superficial urbano contém todos os poluentes que se depositam na superfície do solo, provenientes das atividades urbanas. Já o deflúvio superficial agrícola apresenta características que são influenciadas pelas práticas agrícolas utilizadas em cada região, cuja composição varia de acordo com o período do ano em que se prepara o terreno para o plantio, processa a aplicação de fertilizantes e defensivos, e durante a colheita. ${ }^{4-8}$

Por outro lado, observa-se na calha do Rio Cachoeira um incremento na produção de sedimentos principalmente nas áreas periféricas das grandes cidades (Itabuna e Ilhéus), devido à falta de infraestrutura. Entre os principais problemas causados pelo aumento da produção de sedimentos estão o assoreamento da calha fluvial e o transporte de poluentes associados. Os sedimentos são importantes compartimentos de acúmulo ou liberação de metais para os sistemas aquáticos, possuindo elevada capacidade de retenção e acúmulo de

*e-mail: zesoares@uesb.edu.br elementos traços lançados na coluna d'água. Em consequência, estudos de metais nestes compartimentos são relevantes na avaliação da poluição e contaminação dos recursos hídricos. ${ }^{9-13}$

Para avaliar o papel do sedimento na retenção ou disponibilização de elementos tóxicos para o meio aquoso, devem-se realizar medidas do conteúdo total atrelado ao teor de metais particionado nas diferentes fases do sedimento, visto que a importância toxicológica dos metais depende de sua biodisponibilidade. É possível encontrar elevados teores de um elemento metálico tóxico no sedimento, no entanto sua forma de associação não o disponibiliza facilmente para a biota. O comportamento dos metais depende estritamente de sua forma química. Para uma compreensão dos potenciais de disponibilidade dos metais, devem-se realizar extrações seletivas usando reagentes específicos.

Encontram-se disponíveis na literatura diversos procedimentos de extração sequencial de metais em solos e sedimentos. Tais procedimentos contemplam uma variedade de esquemas de fracionamento, o que indica a inexistência de um método padrão para avaliação da mobilidade metais nesses compartimentos. ${ }^{14-25}$

O método desenvolvido por Tessier et al. ${ }^{14}$ foi considerado adequado para este trabalho, por se tratar de um dos precursores da extração sequencial de metais em solos e sedimentos e por ser um dos métodos mais citados e utilizados nos trabalhos que avaliam a mobilidade de elementos traços em sedimentos de rios. ${ }^{14,26}$ Através desse procedimento de extração sequencial é possível constatar a presença de metais nas formas químicas mais lábeis (como na fase trocável e associada ao carbonato) ou mais estáveis, de menor mobilidade e/ou biodisponibilidade (ligadas às fases de óxido de $\mathrm{Fe}$ e Mn e à matéria orgânica).

A proposta deste trabalho foi avaliar a distribuição de $\mathrm{Zn}, \mathrm{Pb}, \mathrm{Ni}$, $\mathrm{Cu}, \mathrm{Mn}$ e Fe no sedimento superficial do Rio Cachoeira no percurso entre as cidades de Itabuna e Ilhéus na região sul da Bahia, além da influência espacial e temporal na mobilidade dos metais no sedimento, com base no método de fracionamento utilizando a extração sequencial em cinco etapas.

Os seis metais avaliados neste trabalho foram escolhidos com base nos impactos mais relevantes associados às águas e sedimentos do Rio Cachoeira no local em estudo (drenagem dos escoamentos urbanos, industriais e agrícolas). O enriquecimento do $\mathrm{Zn}, \mathrm{Ni}, \mathrm{Pb}$ e 
$\mathrm{Cu}$ nas frações lábeis do sedimentos do Rio poderá ser indicativo da poluição provocada pelo deflúvio urbano e pela drenagem de efluentes industriais. A poluição oriunda das atividades agrícolas poderá ser avaliada pelo enriquecimento do $\mathrm{Ni}, \mathrm{Cu}, \mathrm{Zn}, \mathrm{Mn}$ e Fe nos sedimentos do rio. ${ }^{27}$

\section{PARTE EXPERIMENTAL}

\section{Descrição da área em estudo}

O estudo foi realizado em uma parte do curso do Rio Cachoeira que se estende por uma distância aproximada de $40 \mathrm{~km}$, englobando o perímetro urbano e rural da cidade de Itabuna e chegando até as proximidades do perímetro urbano da cidade de Ilhéus, sendo que os pontos de coleta são mostrados na Figura 1S, material suplementar. O local encontra-se inserido em zona de Mata Atlântica o que denota uma região com altos índices pluviométricos, com períodos de chuva bastante diferenciados, ocorrendo durante todo o ano, porém divididos em período de chuvas intensas (chuvoso) e período de chuvas fracionadas (seco). A faixa de estudo compreendeu a porção da Bacia do Rio Cachoeira próxima ao litoral, com clima quente e úmido e precipitação anual superior a $1000 \mathrm{~mm}$, bem distribuída durante todo o ano

\section{Amostragem}

Os pontos de amostragem foram escolhidos seguindo o critério de representatividade dentro do contexto de contaminação, observando os principais responsáveis pelas possíveis alterações da qualidade dos recursos hídricos em estudo. Cada uma das estações de coleta foi georreferenciada com base no sistema de posicionamento global - GPS (global position system).

A Tabela 1 apresenta a descrição dos pontos e suas características de acordo com as atividades relacionadas com os usos do solo e da água.

As coletas das amostras de sedimento foram realizadas conforme procedimento descrito por Santos et al. ${ }^{28}$ Com base neste procedimento, utilizou-se um artefato de PVC na obtenção das amostras, as quais foram mantidas em temperatura abaixo de $0{ }^{\circ} \mathrm{C}$.

\section{Preparo das amostras de sedimento}

As amostras de sedimento foram secas em estufa durante $24 \mathrm{~h}$ à temperatura de $60^{\circ} \mathrm{C}$. Algumas amostras apresentavam pedaços de madeira e rochas com alta granulometria, que foram então retiradas antes do processo de destorroamento, com posterior peneiração. As frações de cada amostra, após tamisação em peneira de $65 \mu \mathrm{m}$, foram recolhidas e acondicionadas em frascos de polietileno de alta densidade.$^{28,29}$ A seguir, o procedimento de extração seletiva foi aplicado para se avaliar a disponibilidade dos metais.

Neste trabalho foi utilizado um método de extração química que permitiu avaliar a associação dos metais em quatro frações do sedimento: a fração facilmente trocável, a fração ligada a carbonatos, a fração ligada a óxidos de manganês e ferro amorfo facilmente redutível, e a fração ligada a óxidos de ferro e manganês cristalino moderadamente redutível. A escolha dos extratores foi baseada principalmente no trabalho de Bevilacqua et al. ${ }^{26}$ que utilizaram um procedimento derivado do método de Tessier et al.. ${ }^{14}$

Para obtenção da fração trocável (F1), 2,0 g de sedimento foram

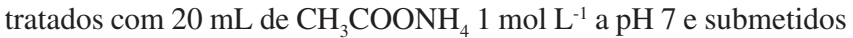
à agitação mecânica por $2 \mathrm{~h}$, em temperatura de $22^{\circ} \mathrm{C}$. A solução sobrenadante foi retirada através de sucção.

A fração ligada a carbonato (F2) foi obtida pela adição de $20 \mathrm{~mL}$ de $\mathrm{CH}_{3} \mathrm{COONa} / \mathrm{CH}_{3} \mathrm{COOH} 1 \mathrm{~mol} \mathrm{~L}^{-1}$ a pH 5 no resíduo obtido na etapa anterior. A mistura foi submetida à agitação por $6 \mathrm{~h}$ a $22{ }^{\circ} \mathrm{C} \mathrm{e}$ a solução sobrenadante foi retirada através de sucção.

A fração ligada a óxidos de manganês e ferro amorfo (F3) foi obtida pela adição de $20 \mathrm{~mL}$ de $\mathrm{NH}_{2} \mathrm{OH}$. $\mathrm{HCl} / \mathrm{CH}_{3} \mathrm{COOH} 0,01 \mathrm{~mol} \mathrm{~L}^{-1}$ a pH 2 no resíduo obtido na etapa anterior. A mistura foi submetida à agitação por $12 \mathrm{~h}$ a $22{ }^{\circ} \mathrm{C}$ e a solução sobrenadante foi retirada através de sucção.

A fração ligada a óxidos de manganês e ferro cristalino (F4) foi obtida pela adição de $20 \mathrm{~mL}$ de $\left(\mathrm{NH}_{4}\right)_{2} \mathrm{C}_{2} \mathrm{O}_{4} / \mathrm{H}_{2} \mathrm{C}_{2} \mathrm{O}_{4} 0,1 \mathrm{~mol} \mathrm{~L}^{-1}$ a pH 3 no resíduo obtido na etapa anterior. A mistura foi submetida à agitação por $24 \mathrm{~h}$ a $22{ }^{\circ} \mathrm{C}$ sob a ação catalítica da luz, a solução sobrenadante foi retirada através de sucção.

A fração residual (FR) dos metais no sedimento foi obtida pela digestão assistida por micro-ondas, baseada no procedimento EPA3051. Adicionaram-se $10 \mathrm{~mL}$ de $\mathrm{HNO}_{3}$ concentrado em $0,5 \mathrm{~g}$ de sedimento. A seguir, submeteu-se à irradiação em forno micro-ondas pressurizado, onde em 5,5 min alcançou a temperatura de $175{ }^{\circ} \mathrm{C}$, que foi mantida por 4,5 min. Separou-se o resíduo remanescente por filtragem em papel quantitativo Whatman, $\mathrm{n}^{\circ} 42$.

\section{Procedimentos analíticos}

As determinações dos elementos $\mathrm{Zn}, \mathrm{Pb}, \mathrm{Ni}, \mathrm{Cu}, \mathrm{Mn}$ e $\mathrm{Fe}$ nas amostras de sedimento foram realizadas empregando-se um espectrômetro de absorção atômica por chama (FAAS), modelo AAnalyst 200 (USA), equipado com corretor de fundo de deutério. A resposta do instrumento foi conferida periodicamente com soluções padrões de concentração conhecida. O sistema de aquisição de dados foi gerenciado por microcomputador acoplado ao espectrômetro de absorção atômica e como fonte de radiação foi empregada lâmpada de cátodo oco dos respectivos metais.

A performance dos procedimentos de extração sequencial e determinação dos analitos foi avaliada com base nos procedimentos apresentado por Santos et.al. ${ }^{28}$ e Silva et al.. ${ }^{25}$ Os valores das concentrações dos

Tabela 1. Pontos de amostragem e suas características

\begin{tabular}{|c|c|c|}
\hline Ponto & Coordenadas eeográficas & Uso de solo \\
\hline P1 & 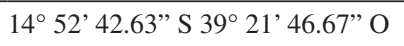 & Área rural, a montante do centro urbano da cidade de Itabuna (agricultura e pecuária) \\
\hline $\mathrm{P} 2$ & $14^{\circ} 48^{\prime} 51.88^{\prime \prime} \mathrm{S} 39^{\circ} 17^{\prime} 43.15^{\prime \prime} \mathrm{O}$ & Centro urbano da cidade de Itabuna, drenagem de efluentes industriais e domésticos \\
\hline P3 & $14^{\circ} 47^{\prime} 20.22^{\prime \prime} \mathrm{S} 39^{\circ} 15^{\prime} 9.79^{\prime \prime} \mathrm{O}$ & Área semiurbana, localizada após o centro urbano da cidade de Itabuna \\
\hline P4 & $14^{\circ} 47^{\prime} 20.22 ” \mathrm{~S} 39^{\circ} 15^{\prime} 9.79 ” \mathrm{O}$ & Agricultura, pecuária e drenagem de efluentes domésticos \\
\hline P5 & $14^{\circ} 47^{\prime} 52.49^{\prime \prime} \mathrm{S} 39^{\circ} 12^{\prime} 48.06^{\prime \prime} \mathrm{O}$ & Agricultura, pecuária e drenagem de efluentes domésticos \\
\hline P6 & $14^{\circ} 47^{\prime} 43.44^{\prime \prime} \mathrm{S} 39^{\circ} 11^{\prime} 3.54^{\prime \prime} \mathrm{O}$ & Agricultura, pecuária e drenagem de efluentes do centro de pesquisa (CEPLAC) \\
\hline P7 & $14^{\circ} 48^{\prime} 24.37^{\prime \prime} \mathrm{S} 39^{\circ} 8^{\prime} 54.04^{\prime \prime} \mathrm{O}$ & Agricultura, pecuária e drenagem de efluentes da UESC e de uma fábrica de aromatizantes \\
\hline P8 & $14^{\circ} 47^{\prime} 9.74^{\prime \prime} \mathrm{S} 39^{\circ} 5^{\prime} 31.81^{\prime \prime} \mathrm{O}$ & Agricultura, pecuária e drenagem de efluentes municipais \\
\hline P9 & $14^{\circ} 47^{\prime} 58.62^{\prime \prime} \mathrm{S} 39^{\circ} 5 ’ 1.42^{\prime \prime} \mathrm{O}$ & $\begin{array}{l}\text { Agricultura, pecuária e drenagem de efluentes da ETE da cidade de Ilhéus, este ponto localiza-se próximo } \\
\text { ao estuário }\end{array}$ \\
\hline
\end{tabular}


analitos obtidos nas etapas da extração sequencial, incluindo a fração residual (F1 + F2 + F3 + F4 + FR), foram comparados com os valores obtidos pela digestão ácida assistida por micro-ondas nas mesmas amostras de sedimento. Os sedimentos oriundos dos 9 pontos de coleta apresentaram recuperações médias que variaram de 92 a 104\% nas amostras coletadas no período chuvoso e de 89 a $108 \%$ nas amostras do período seco, indicando que a soma dos valores das concentrações dos analitos determinados nas frações da extração sequencial corresponde aos teores dos metais totais obtidos pela digestão ácida.

\section{RESULTADOS E DISCUSSÃO}

\section{Padrão de distribuição dos metais totais nos sedimentos}

A Tabela 2 apresenta as concentrações médias de $\mathrm{Zn}, \mathrm{Pb}, \mathrm{Ni}$, $\mathrm{Cu}, \mathrm{Mn}$ e Fe na fração residual do sedimento coletado em novembro de 2008 (período seco) e abril de 2009 (período chuvoso). Pode-se observar que os metais $\mathrm{Zn}, \mathrm{Pb}$ e $\mathrm{Cu}$ apresentaram as concentrações médias mais elevadas nas amostras dos 3 primeiros pontos $(\mathrm{P} 1, \mathrm{P} 2 \mathrm{e}$ P3), coletadas no período seco. Deve-se destacar que, entre os dois primeiros pontos de coleta (P1 e P2) existe um importante parque industrial, cujas indústrias lançam seus efluentes diretamente no Rio Cachoeira, podendo influenciar nos teores desses elementos nos sedimentos na faixa que abrange esses dois pontos de coleta. Já na faixa que abrange o ponto $\mathrm{P} 3$, o aumento dos teores de $\mathrm{Zn}, \mathrm{Pb}$ e $\mathrm{Cu}$ no período seco pode ser atribuído à influência das atividades urbanas, tendo em vista que nesse percurso o Rio Cachoeira começa a receber os despejos de esgoto sanitário e águas pluviais drenados por um sistema de coleta combinado através de galerias e emissários.
Após o ponto $\mathrm{P} 3$ a atividade agrícola é a principal fonte de efluentes lançados no rio destacando-se a cultura do cacau, cujo manejo se utiliza de elevadas aplicações de fertilizantes inorgânicos. Esses efluentes provavelmente estão contribuindo para o aumento dos teores de $\mathrm{Zn}$, Ni e Cu no sedimento na faixa que abrange os pontos P5 e P6.

Por outro lado, os aportes de efluentes oriundos das atividades industriais, urbanas e agrícolas refletiram nos elevados valores de matéria orgânica nos sedimentos coletados nos pontos de amostragem P1, P2, P3 e P6, respectivamente. Os sedimentos oriundos desses pontos apresentaram teores de matéria orgânica (MO) que variaram entre 6,50 a 8,13\%, enquanto que nos sedimentos coletados nos demais pontos (P4, P5, P7, P8 e P9) os teores de matéria orgânica variaram entre 3,10 a 4,02\%. Os valores de $\mathrm{pH}$ medidos nas águas durante a coleta ficaram situados entre 6,3 a 7,1 na estação seca e 6,7 a 7,7 na estação chuvosa.

Sabe-se que os íons metálicos nos sedimentos de fundo podem se ligar aos sítios ativos das moléculas orgânicas ou podem ser precipitados como sulfetos. Devido à redução dos teores de oxigênio dissolvido (OD), provocada pelas atividades microbianas no sedimento, o sulfato torna-se o principal aceptor de elétrons na oxidação da matéria orgânica. O sulfeto gerado, denominado de AVS (acid volatile sulfide), pode formar um sulfeto metálico estável durante o processo da oxidação da MO no sedimento. Deste modo, a formação do AVS pode governar o comportamento do íon metálico divalente nos sedimentos ricos em matéria orgânica. Esse fato também pode explicar os elevados valores nas concentrações de $\mathrm{Zn}, \mathrm{Pb}$ e $\mathrm{Cu}$ nos três primeiros pontos de coleta (P1, P2 e P3).

A Tabela 2, além das concentrações médias dos metais avaliados, mostra os valores de TEC e PEC. No período chuvoso, com exceção

Tabela 2. Concentração média de $\mathrm{Zn}, \mathrm{Pb}, \mathrm{Ni}, \mathrm{Cu}, \mathrm{Mn}$ e Fe em $\mathrm{mg} \mathrm{kg}^{-1} \pm$ desvio padrão, medidos na fração residual das amostras de sedimento

\begin{tabular}{|c|c|c|c|c|c|c|}
\hline & $\mathrm{Zn}\left(\mathrm{mg} \mathrm{kg}^{-1}\right)$ & $\mathrm{Pb}\left(\mathrm{mg} \mathrm{kg}^{-1}\right)$ & $\mathrm{Ni}\left(\mathrm{mg} \mathrm{kg}^{-1}\right)$ & $\mathrm{Cu}\left(\mathrm{mg} \mathrm{kg}^{-1}\right)$ & $\operatorname{Mn}\left(\mathrm{mg} \mathrm{kg}^{-1}\right)$ & $\mathrm{Fe}\left(\mathrm{mg} \mathrm{kg}^{-1}\right)$ \\
\hline \multicolumn{7}{|l|}{$\underline{\text { Nov} / 2008}$} \\
\hline $\mathrm{P} 1$ & $128,9 \pm 1,5$ & $29,9 \pm 1,5$ & $<0,047^{\hat{a}}$ & $206,3 \pm 9,8$ & $40,3 \pm 1,8$ & $3626 \pm 137$ \\
\hline $\mathrm{P} 2$ & $268,3 \pm 9,1$ & $58,5 \pm 2,3$ & $<0,047^{\hat{a}}$ & $179,3 \pm 3,2$ & $76,9 \pm 3,3$ & $13484 \pm 564$ \\
\hline P3 & $152,8 \pm 2,8$ & $25,7 \pm 2,8$ & $<0,047^{\hat{a}}$ & $84,0 \pm 7,1$ & $250,8 \pm 6,0$ & $22354 \pm 123$ \\
\hline $\mathrm{P} 4$ & $39,0 \pm 3,2$ & $<0,060^{\hat{a}}$ & $12,9 \pm 0,8$ & $14,9 \pm 2,2$ & $225,3 \pm 9,5$ & $20246 \pm 504$ \\
\hline P5 & $84,6 \pm 4,9$ & $12,6 \pm 0,9$ & $32,8 \pm 3,1$ & $77,1 \pm 3,9$ & $170,9 \pm 5,3$ & $22447 \pm 268$ \\
\hline P6 & $122,8 \pm 8,6$ & $<0,060^{\hat{a}}$ & $28,2 \pm 4,5$ & $95,3 \pm 4,9$ & $450,5 \pm 2,1$ & $33238 \pm 284$ \\
\hline P7 & $55,8 \pm 1,2$ & $11,3 \pm 0,8$ & $10,4 \pm 1,0$ & $81,9 \pm 3,5$ & $400,3 \pm 12,4$ & $17251 \pm 264$ \\
\hline P8 & $85,0 \pm 5,4$ & $<0,060^{\mathrm{a}}$ & $15,4 \pm 0,5$ & $24,5 \pm 1,9$ & $176,8 \pm 7,6$ & $28401 \pm 170$ \\
\hline P9 & $47,7 \pm 1,7$ & $<0,060^{\hat{a}}$ & $22,9 \pm 0,8$ & $14,1 \pm 1,6$ & $49,9 \pm 2,3$ & $14790 \pm 180$ \\
\hline \multicolumn{7}{|l|}{$\underline{\mathrm{Abr} / 2009}$} \\
\hline $\mathrm{P} 1$ & $15,0 \pm 0,1$ & $3,4 \pm 0,3$ & $3,7 \pm 0,2$ & $8,9 \pm 0,8$ & $169,9 \pm 6,5$ & $5927 \pm 77$ \\
\hline $\mathrm{P} 2$ & $41,1 \pm 0,1$ & $<0,060^{\hat{a}}$ & $5,7 \pm 0,1$ & $18,3 \pm 0,3$ & $89,0 \pm 3,6$ & $5177 \pm 73$ \\
\hline P3 & $35,1 \pm 0,1$ & $<0,060^{\hat{a}}$ & $4,7 \pm 0,1$ & $9,7 \pm 0,2$ & $110,4 \pm 3,2$ & $8978 \pm 69$ \\
\hline $\mathrm{P} 4$ & $13,5 \pm 0,1$ & $<0,060^{\mathrm{a}}$ & $4,1 \pm 0,2$ & $7,3 \pm 0,2$ & $91,1 \pm 3,5$ & $3669 \pm 22$ \\
\hline P5 & $12,1 \pm 0,1$ & $<0,060^{\hat{a}}$ & $2,6 \pm 0,3$ & $4,9 \pm 0,1$ & $124,0 \pm 3,3$ & $6344 \pm 159$ \\
\hline P6 & $39,4 \pm 0,1$ & $<0,0^{6} 0 \mathrm{a}$ & $29,7 \pm 0,2$ & $74,7 \pm 0,3$ & $93,2 \pm 1,7$ & $22637 \pm 63$ \\
\hline P7 & $15,3 \pm 0,1$ & $32,0 \pm 1,2$ & $10,0 \pm 0,2$ & $12,8 \pm 0,3$ & $94,8 \pm 2,3$ & $4205 \pm 95$ \\
\hline P8 & $46,7 \pm 0,1$ & $14,2 \pm 3,3$ & $12,4 \pm 0,1$ & $16,3 \pm 0,2$ & $361,0 \pm 6,0$ & $12212 \pm 66$ \\
\hline P9 & $34,2 \pm 0,1$ & $<0,060^{\hat{a}}$ & $13,9 \pm 0,2$ & $16,9 \pm 0,2$ & $113,0 \pm 3,0$ & $14449 \pm 108$ \\
\hline TEC $\left(\mathrm{mg} \mathrm{kg}^{-1}\right)$ & 120,0 & 36,00 & 23,00 & 32,00 & 460,0 & 20000 \\
\hline PEC $\left(\mathrm{mg} \mathrm{kg}^{-1}\right)$ & 460,0 & 130,0 & 49,00 & 150,0 & 1100 & 40000 \\
\hline
\end{tabular}

*Limite de quantificação (LQ): calculado como 10 vezes o desvio padrão de 12 extrações do branco, dividido pela inclinação da curva de calibração. 
do $\mathrm{Ni}, \mathrm{Cu}$ e Fe no ponto P6, as concentrações dos metais nas demais amostras de sedimento apresentaram-se abaixo do valor TEC, não devendo provocar efeitos adversos para os organismos aquáticos neste período. Já no período seco, apenas o Mn não apresentou valores de concentração acima do TEC em nenhuma amostra de sedimentos. Neste período os valores de concentração do Zn excederam o TEC nos pontos $\mathrm{P} 1, \mathrm{P} 2$, P3 e P6, o Pb no ponto $\mathrm{P} 2$, o Ni nos pontos P5 e P6 e o Fe nos pontos P3, P4, P5 e P8. Entre os elementos avaliados pode-se destacar o $\mathrm{Cu}$, que em apenas três pontos de coleta (P4, P8 e P9) não apresentou valores de concentração acima do TEC nos sedimentos, sendo que nos pontos $\mathrm{P} 1$ e P2 as concentrações situaram-se acima do valor PEC. Portanto, fica evidente que no período seco os metais avaliados apresentam muito mais efeitos adversos para os organismos no sedimento do Rio Cachoeira do que no período chuvoso.

\section{Teores de $\mathrm{Zn}, \mathrm{Pb}, \mathrm{Ni}, \mathrm{Cu}$, Mn e Fe nas frações do sedimento}

A compreensão da distribuição espacial e temporal dos teores destes metais nas diferentes fases do sedimento é muito importante para se entender a forma pela qual se encontram associados e, assim, indicar sua mobilidade e os fatores que estão influenciando essas mudanças. Os resultados das concentrações relativas de $\mathrm{Zn}, \mathrm{Pb}, \mathrm{Ni}, \mathrm{Cu}$, Mn e Fe em cada uma das frações dos sedimentos são apresentados nas Figuras $2 \mathrm{~S}$ e $3 \mathrm{~S}$, material suplementar.

A Figura $2 \mathrm{~S}$, material suplementar, mostra que o Fe se encontra mais associado à fração residual (FR) do sedimento. No ponto P6 (período chuvoso) e nos pontos $\mathrm{P} 4, \mathrm{P} 8$ e P9 (período seco) esse elemento se apresentou com mais de $80 \%$ de sua concentração na fração residual do sedimento. Os teores de $\mathrm{Fe}$ associados às frações não residuais ou lábeis do sedimento foram encontrados principalmente na fração redutível (óxidos e hidróxidos cristalinos), nos dois períodos de amostragem. Ao longo da área em estudo, o Mn apresentou uma afinidade forte com as frações trocáveis $(\mathrm{F} 1)$ e ligadas a carbonato (F2), visto que nos pontos $\mathrm{P} 1, \mathrm{P} 2, \mathrm{P} 3$ e $\mathrm{P} 4$, nos dois períodos de amostragem, essas duas frações $(\mathrm{F} 1+\mathrm{F} 2)$ representaram em torno de $40 \%$ do conteúdo de manganês total no sedimento.

Na Figura 3S, material suplementar, pode-se observar que teores significativos de $\mathrm{Ni}$ e $\mathrm{Cu}$ se encontram adsorvidos nos óxidos cristalinos ou moderadamente redutíveis de Fe e Mn (fração F4) tanto no período chuvoso quanto no período seco. No período seco o Ni foi encontrado associado à fração facilmente trocável (F1) somente nos pontos P8 e P9.

No caso do Zn observou-se uma distribuição semelhante em três frações lábeis (ligada a carbonato, adsorvida a óxidos facilmente redutível de Fe e Mn e adsorvida a óxidos cristalinos ou moderadamente redutíveis de $\mathrm{Fe}$ ), nos dois períodos de amostragem. Entretanto, no ponto P5 é importante salientar a ocorrência de uma fração significativa do $\mathrm{Zn}$ associada à fase facilmente trocável no período chuvoso. Deve-se destacar que os sedimentos dessa zona de amostragem sofrem influência das atividades agrícolas, que utilizam $\mathrm{Zn}$ como nutriente.

Para os pontos que recebem uma maior influência das atividades urbanas (P2 e P3) a Figura 3S, material suplementar, mostra que os padrões de distribuição do $\mathrm{Cu}$ nas frações lábeis, no período chuvoso, apresentaram características bem diferentes quando comparados com os pontos que se localizam fora do perímetro urbano da cidade de Itabuna. Isto porque a soma dos teores de cobre distribuídos entre as frações lábeis $(\mathrm{F} 1+\mathrm{F} 2+\mathrm{F} 3+\mathrm{F} 4)$ dessas amostras de sedimento foi de aproximadamente $50 \%$. Tal fato pode ser atribuído ao grande volume de chuvas ocorrido na região no período que antecedeu a coleta (em torno de 504,1 $\mathrm{mm}$ ) que pode ter provocado a drenagem de resíduos de cobre oriundos das atividades urbanas.

$\mathrm{O} \mathrm{Ni}$ apresentou as maiores concentrações na fração disponível das amostras coletadas no período seco nos cinco primeiros pontos de coleta (P1, P2, P3, P4 e P5), incluindo os pontos localizados no perímetro urbano. Deve-se destacar que no período chuvoso, esse elemento não foi detectado em nenhuma das frações do sedimento nos três primeiros pontos de coleta (P1, P2 e P3).

Nos sedimentos coletados nos dois períodos (seco e chuvoso) não foram detectados teores de $\mathrm{Pb}$ nas frações lábeis, exceto nas amostras de sedimento coletadas no período chuvoso nos pontos P3 e P5, onde foram detectados teores desse elemento na fração trocável (F1) em percentuais extremamente baixos.

\section{Propriedades físico-químicas e o comportamento dos metais na coluna d'água e nas frações do sedimento}

A importância de identificar os níveis de associação dos metais nas frações do sedimento pode ser ilustrada com base na mobilidade do Ni no ambiente em estudo.

Entre os metais avaliados nas amostras de água coletadas nos mesmos locais onde foram amostrados os sedimentos, apenas o níquel apresentou valores de concentração acima do limite de quantificação (LQ) do método $\left(47 \mu \mathrm{g} \mathrm{L}^{-1}\right)$. Os resultados das concentrações de $\mathrm{Ni}$ nas águas e nas frações (F1, F2, F3 e F4) dos sedimentos e as medidas de $\mathrm{pH}$, condutividade elétrica (CE), sólidos totais dissolvidos (STD) nas amostras de água estão representados na Tabela 3.

Com base nas características físico-químicas das águas do Rio Cachoeira, os resultados indicam que a região que abrange os pontos P8 e P9 apresenta características similares, mas bem diferenciadas quando comparadas com os demais pontos de coleta. Nos dois períodos de amostragem (seco e chuvoso), as águas coletadas nesses dois pontos apresentaram valores de CE e STD bem maiores quando comparados com aqueles apresentados pelas amostras coletadas nos demais pontos. Essa diferença se deve ao fato desses pontos localizarem-se nas proximidades da região estuarina formada pelo encontro do Rio Cachoeira com o Oceano Atlântico, na cidade de Ilhéus.

Entre as características físico-químicas, o pH é considerado a variável de maior impacto na mobilização dos elementos traços retidos no sedimento. ${ }^{30}$ Os reagentes utilizados no processamento das duas primeiras frações (F1 e F2) provocam a liberação dos metais pela redução do $\mathrm{pH}$ do meio. Entretanto, as águas dos dois períodos de amostragens apresentaram valores de $\mathrm{pH}$ ao redor da neutralidade, variando de 6,33 a 7,12 no período seco e de 6,33 a 7,66 no período chuvoso. Pode-se concluir que esta variável não pode ser considerada como indicativa da mobilidade dos metais no ambiente aquático em estudo.

Com relação ao níquel, observa-se um aumento progressivo de sua concentração entre os pontos de coleta $\mathrm{P} 1$ a P9, nas amostras coletadas em novembro de 2008 (período seco), cujas concentrações variaram entre 60 a $447 \mu \mathrm{g} \mathrm{L}{ }^{-1}$. Pode-se destacar que a drenagem de efluentes industriais provavelmente influenciou nas maiores concentrações de Ni detectadas nas amostras coletadas no ponto P2 $\left(100 \mu \mathrm{g} \mathrm{L}^{-1}\right)$ em comparação com as encontradas nos dois pontos subsequentes P3 e P4 (70 e $84 \mu \mathrm{g} \mathrm{L}^{-1}$, respectivamente). Os resultados da Tabela 3 evidenciam também, um aumento acentuado dos teores de Ni nos pontos $\mathrm{P} 8$ e $\mathrm{P} 9$ neste período.

Ao contrário do que ocorreu na primeira amostragem, os teores de Ni nas amostras coletadas no período chuvoso (abril de 2009) apresentaram-se concordantes em todos os pontos da área em estudo. Neste período, devido à movimentação das águas provocada pelos elevados índices pluviométricos, deve ter ocorrido em alguma extensão a homogeneização dos metais dissolvidos nas águas do Rio Cachoeira.

Deve-se ressaltar que as águas dos dois períodos de coleta apresentaram valores de concentração do níquel acima daqueles estabelecido pelos órgãos oficiais para águas naturais. A resolução CONAMA n ${ }^{\circ} 357$, de 2005, estabelece uma concentração máxima de 
Tabela 3. Concentração média de Ni nas águas e nas frações (F1, F2, F3 e F4) dos sedimentos e valores de pH, STD e CE das amostras de água do Rio Cachoeira, coletadas em novembro de 2008 e abril de 2009

\begin{tabular}{|c|c|c|c|c|c|c|c|c|c|}
\hline Parâmetros & $\mathrm{P} 1$ & P2 & P3 & P4 & P5 & P6 & P7 & P8 & P9 \\
\hline \multicolumn{10}{|l|}{ Nov/2008 } \\
\hline $\mathrm{pH}$ & 6,96 & 6,68 & 6,33 & 6,38 & 6,71 & 6,77 & 7,13 & 6,76 & 6,67 \\
\hline STD $\left(\mathrm{mg} \mathrm{L}^{-1}\right)$ & 221 & 366 & 324 & 314 & 270 & 293 & 285 & 10466 & 10486 \\
\hline $\mathrm{CE}\left(\mu \mathrm{S} \mathrm{cm} \mathrm{cm}^{-1}\right)$ & 661 & 980 & 745 & 710 & 575 & 731 & 715 & 20050 & 20926 \\
\hline $\mathrm{Ni}\left(\mu \mathrm{g} \mathrm{L}{ }^{-1}\right)$ & $60 \pm 1$ & $100 \pm 8$ & $70 \pm 4$ & $84 \pm 4$ & $121 \pm 4$ & $113 \pm 9$ & $196 \pm 3$ & $437 \pm 8$ & $447 \pm 9$ \\
\hline $\mathrm{Ni}\left(\mu \mathrm{g} \mathrm{L}^{-1}\right)^{\mathrm{F} 1}$ & $<\mathrm{LQ}$ & $<\mathrm{LQ}$ & $<\mathrm{LQ}$ & $57 \pm 2$ & $<\mathrm{LQ}$ & $1590 \pm 9$ & $1050 \pm 2$ & $1650 \pm 7$ & $1260 \pm 9$ \\
\hline $\mathrm{Ni}\left(\mu \mathrm{g} \mathrm{L}^{-1}\right)^{\mathrm{F} 2}$ & $<\mathrm{LQ}$ & $<\mathrm{LQ}$ & $<\mathrm{LQ}$ & $2180 \pm 2$ & $3060 \pm 2$ & $2470 \pm 6$ & $2100 \pm 5$ & $1770 \pm 2$ & $1630 \pm 5$ \\
\hline $\mathrm{Ni}\left(\mu \mathrm{g} \mathrm{L}^{-1}\right)^{\mathrm{F} 3}$ & $<\mathrm{LQ}$ & $<\mathrm{LQ}$ & $<\mathrm{LQ}$ & $1650 \pm 3$ & $2000 \pm 2$ & $2110 \pm 3$ & $1790 \pm 2$ & $1650 \pm 4$ & $958 \pm 6$ \\
\hline $\left.\mathrm{Ni}(\mu \mathrm{g} \mathrm{L})^{-1}\right)^{\mathrm{F} 4}$ & $<\mathrm{LQ}$ & $<\mathrm{LQ}$ & $<\mathrm{LQ}$ & $6075 \pm 9$ & $8050 \pm 17$ & $8610 \pm 9$ & $3210 \pm 2$ & $4420 \pm 6$ & $4690 \pm 11$ \\
\hline \multicolumn{10}{|l|}{ Abr/2009 } \\
\hline $\mathrm{pH}$ & 7,66 & 7,15 & 6,92 & 7,62 & 6,76 & 6,77 & 7,22 & 6,74 & 6,79 \\
\hline $\mathrm{STD}\left(\mathrm{mg} \mathrm{L}^{-1}\right)$ & 525 & 336 & 320 & 209 & 190 & 211 & 201 & 10313 & 10332 \\
\hline $\mathrm{CE}\left(\mu \mathrm{S} \mathrm{cm}^{-1}\right)$ & 466 & 609 & 490 & 421 & 379 & 571 & 560 & 19606 & 20124 \\
\hline $\mathrm{Ni}\left(\mu \mathrm{g} \mathrm{L}^{-1}\right)$ & $218 \pm 1$ & $174 \pm 3$ & $108 \pm 1$ & $188 \pm 8$ & $216 \pm 4$ & $270 \pm 3$ & $266 \pm 5$ & $296 \pm 5$ & $317 \pm 7$ \\
\hline $\mathrm{Ni}\left(\mu \mathrm{g} \mathrm{L^{-1 }}\right)^{\mathrm{F} 1}$ & $<\mathrm{LQ}$ & $<\mathrm{LQ}$ & $<\mathrm{LQ}$ & $<\mathrm{LQ}$ & $<\mathrm{LQ}$ & $<\mathrm{LQ}$ & $<$ LQ & $240 \pm 10$ & $1000 \pm 10$ \\
\hline $\mathrm{Ni}\left(\mu \mathrm{g} \mathrm{L}^{-1}\right)^{\mathrm{F} 2}$ & $1170 \pm 11$ & $890 \pm 6$ & $730 \pm 8$ & $410 \pm 3$ & $<\mathrm{LQ}$ & $<\mathrm{LQ}$ & $1420 \pm 9$ & $1180 \pm 4$ & $968 \pm 7$ \\
\hline $\mathrm{Ni}\left(\mu \mathrm{g} \mathrm{L}^{-1}\right)^{\mathrm{F} 3}$ & $<\mathrm{LQ}$ & $<\mathrm{LQ}$ & $<\mathrm{LQ}$ & $<\mathrm{LQ}$ & $<\mathrm{LQ}$ & $<\mathrm{LQ}$ & $<\mathrm{LQ}$ & $<\mathrm{LQ}$ & $<\mathrm{LQ}$ \\
\hline $\mathrm{Ni}\left(\mu \mathrm{g} \mathrm{L}^{-1}\right)^{\mathrm{F} 4}$ & $2740 \pm 17$ & $3580 \pm 9$ & $2850 \pm 5$ & $2790 \pm 8$ & $2320 \pm 11$ & $9710 \pm 9$ & $2100 \pm 7$ & $3730 \pm 3$ & $3520 \pm 4$ \\
\hline
\end{tabular}

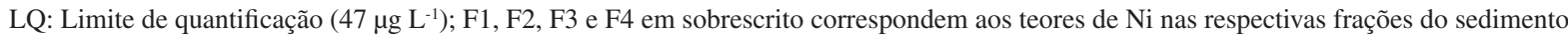

Ni que varia entre 25 a $74 \mu \mathrm{g} \mathrm{L}^{-1}$ para as diversas classes de águas. ${ }^{31}$ Por outro lado, os elevados teores de Ni detectados no Rio Cachoeira apresentam-se coerentes com alguns trabalhos existentes na literatura para águas superficiais. ${ }^{32}$

A Tabela 3 apresenta também os resultados da distribuição Ni nas frações (F1, F2, F3 e F4) do sedimento. No período seco não foram detectados teores de $\mathrm{Ni}$ em nenhuma das quatro frações dos sedimentos coletados nos pontos P1, P2 e P3, situados em áreas influenciadas pelas atividades urbanas. Entretanto, no período chuvoso foram detectadas concentrações significativas de Ni nas frações ligada a carbonato (F2) e ligada a óxidos de manganês e ferro cristalino (F4) nos pontos da área urbana. Este fato pode ser indicativo de um processo de assoreamento da calha do rio com material urbano, que ocorre no período chuvoso.

\section{Análise de agrupamento}

A análise de cluster foi utilizada para avaliar os fatores que afetam a mobilidade dos metais nos dois períodos de amostragem. Para definir a similaridade entre as amostras, os teores de $\mathrm{Zn}, \mathrm{Pb}$, $\mathrm{Ni}, \mathrm{Cu}, \mathrm{Mn}$ e Fe obtidos das frações dos sedimentos e os valores de $\mathrm{pH}$, condutividade elétrica (CE) e sólidos totais dissolvidos (STD) medidos nas águas durante a coleta dos sedimentos foram processados utilizando o agrupamento Ward's method. As medidas de CE e STD nas águas foram consideradas para discriminar os grupos de amostras influenciados pela mudança da atividade iônica provocada pela mistura de águas oceânicas no Rio Cachoeira. A variação desses parâmetros nas águas do Rio Cachoeira na região estuarina poderá ser extremamente importante na avaliação do efeito da salinidade na mobilidade dos metais nos sedimentos em estudo.

Preliminarmente, os valores das variáveis $\mathrm{Zn}, \mathrm{Pb}, \mathrm{Ni}, \mathrm{Cu}, \mathrm{Mn}$, Fe, pH, CE e STD foram normalizados através da transformação
Z-escores, com o objetivo de atribuir um mesmo peso a cada uma delas. Na matriz inicial, os coeficientes de similaridade representam o grau de semelhança entre os pares de amostras e as mesmas foram arranjadas de acordo com os respectivos graus de similaridade, de modo que ficassem agrupadas segundo uma disposição hierárquica.

Nos dendrogramas das Figuras 1a e 1b, as frações residuais (FR) dos sedimentos coletados nos dois períodos formaram agrupamentos distintos a um nível de distância de 25. Em ambos os dendrogramas observaram-se também agrupamentos das amostras coletadas nos pontos $\mathrm{P} 8$ e P9. Esses pontos de amostragem se caracterizam por se localizarem na zona estuarina, onde ocorre o encontro das águas do Rio Cachoeira com as águas do Oceano. Os íons metálicos adsorvidos nas suspensões estáveis da água do Rio Cachoeira, de menor força iônica, são floculados ao encontrar a água do mar, de maior força iônica, arrastando para os sedimentos de fundo os metais presentes na suspensão do rio.

No dendrograma formado pelas variáveis, Figura 1c, é nítida a discriminação dos agrupamentos das variáveis físico-químicas (pH, CE e STD) com os agrupamentos dos teores dos metais ( $\mathrm{Zn}$, $\mathrm{Pb}, \mathrm{Ni}, \mathrm{Cu}, \mathrm{Mn}$ e $\mathrm{Fe}$ ). Entre os agrupamentos formados pelos teores dos metais é possível evidenciar: um formado por $\mathrm{Zn}, \mathrm{Pb}$ e $\mathrm{Cu}$ e outro formado por $\mathrm{Ni}, \mathrm{Fe}$ e $\mathrm{Mn}$. Com isso pode-se sugerir que o $\mathrm{Ni}$ é caracterizado como o metal mais influenciado pela presença dos óxidos e hidróxidos de Fe e Mn nos sedimentos.

\section{CONCLUSÕES}

Os resultados mostram que na fração residual, as concentrações mais elevadas para o $\mathrm{Zn}, \mathrm{Pb}$ e $\mathrm{Cu}$ foram detectadas nos sedimentos oriundos de áreas que sofrem influência das atividades industriais e urbanas. No período seco os teores de $\mathrm{Zn}$ excederam o valor de referência TEC nos sedimentos coletados nos pontos P1, P2, P3 e 

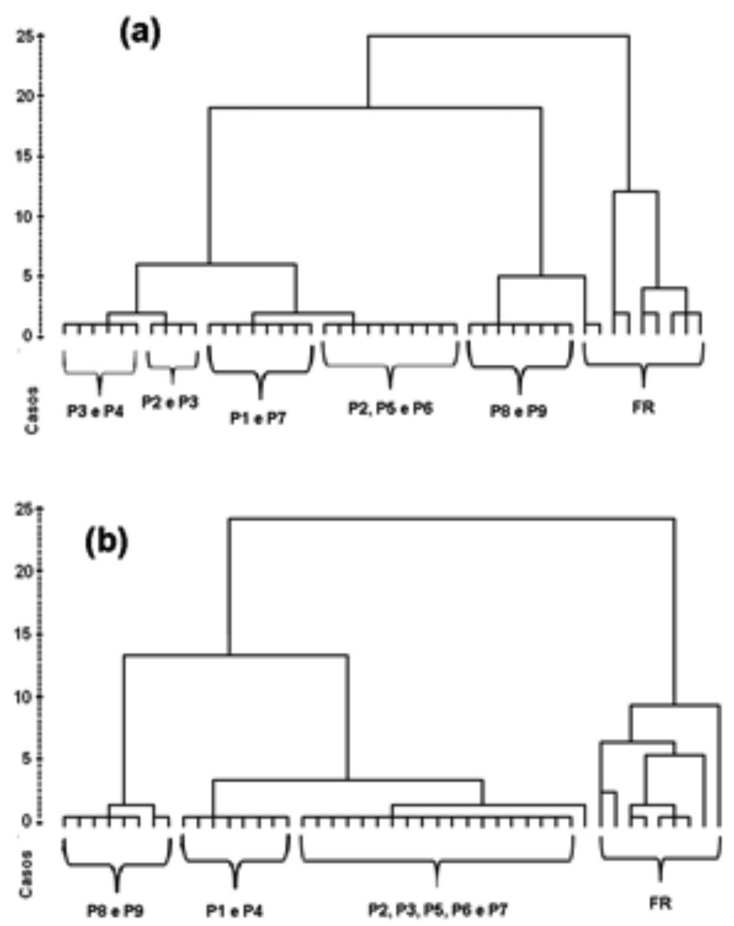

(c)

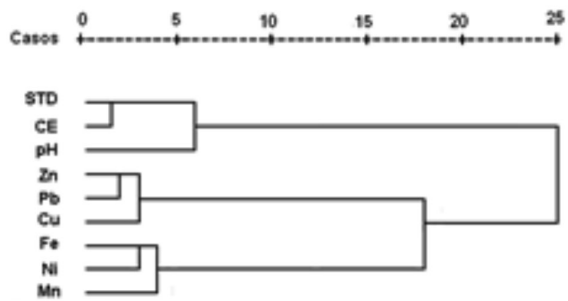

Figura 1. Dendrograma de análise de cluster agrupados pelos resultados das amostras de sedimento coletadas nos dois períodos: (a) período seco; (b) período chuvoso; (c) agrupamento entre variáveis

P6. O Pb excedeu do TEC no ponto P2, o Ni nos pontos P5 e P6 e o Fe nos pontos P3, P4, P5 e P8. Entre os elementos avaliados pode-se destacar o $\mathrm{Cu}$, que em apenas três pontos de coleta (P4, P8 e P9) não apresentou valores de concentração acima do valor de referência TEC nos sedimentos, sendo que nos pontos P1 e P2 as concentrações se situaram acima do valor PEC.

O método de extração química utilizado mostrou-se adequado na caracterização do sedimento e na forma pela qual os metais estão associados. Constatou-se que a maior parte das frações lábeis do $\mathrm{Ni}$ e do $\mathrm{Cu}$ se encontram adsorvidas nos óxidos cristalinos ou moderadamente redutíveis de Fe e Mn (fração F4) tanto no período chuvoso quanto no seco. No caso do Zn observou-se no ponto P5 a existência de uma fração significativa desse elemento associada à fase facilmente trocável no período chuvoso. Deve-se destacar que os sedimentos dessa zona de amostragem sofrem influência das atividades agrícolas, que utilizam o $\mathrm{Zn}$ como nutriente.

Com relação aos possíveis riscos à saúde da população que poderiam ser provocados pelos elementos considerados tóxicos $(\mathrm{Zn}, \mathrm{Cu}$, $\mathrm{Ni}$ e $\mathrm{Pb}$ ), podem-se destacar os teores de $\mathrm{Ni}$ detectados na coluna d'água, acima dos valores estabelecidos pela resolução CONAMA $\mathrm{n}^{\circ} 357$ de 2005. ${ }^{31}$ Águas servidas com elevados teores de Ni podem causar dermatites e afetar os sistemas cardíaco e respiratório. O Zn e o $\mathrm{Cu}$, detectados nas frações mais lábeis do sedimento, também podem ser considerados como potenciais fatores de risco à saúde da população. Uma água de consumo contendo elevados teores de $\mathrm{Cu}$ pode provocar nos indivíduos irritação e corrosão da mucosa, danos capilares generalizados, problemas hepáticos e renais e irritação do sistema nervoso central seguido de depressão.

Entre os elementos tóxicos detectados nos sedimentos, o chumbo é considerado de maior impacto à saúde humana. Uma intoxicação crônica por este metal pode levar a uma doença denominada saturnismo, sendo altamente prejudicial ao desenvolvimento infantil. ${ }^{33}$ Felizmente, a mobilidade desse elemento na biota aquática é baixa, por se encontrar associado quase que exclusivamente à fração residual do sedimento.

\section{MATERIAL SUPLEMENTAR}

No material suplementar, disponível gratuitamente em http:// quimicanova.sbq.org.br na forma de arquivo PDF, com acesso livre, encontram-se os seguintes dados: mapa da área em estudo (Figura 1S) e os resultados das concentrações relativas de $\mathrm{Zn}, \mathrm{Pb}, \mathrm{Ni}, \mathrm{Cu}$, $\mathrm{Mn}$ e Fe em cada uma das frações dos sedimentos, apresentados nas Figuras $2 \mathrm{~S}$ e $3 \mathrm{~S}$.

\section{REFERÊNCIAS}

1. Trindade, M. E. J.; Dissertação de Mestrado, Universidade Estadual de Santa Cruz, Brasil, 2008.

2. Esteves, F. A.; Fundamentos de Limonologia, $2^{\text {a }}$ ed., Interciência: Rio de Janeiro, 1988.

3. Souza, F. M.; Dissertação de Mestrado, Universidade Estadual do Sudoeste da Bahia, Brasil, 2010.

4. Göbel, P.; Dierkes, C.; Coldewey, W. G.; J. Contam. Hydrol. 2007, 91, 26.

5. Chalmers, A. T.; van Metre, P. C.; Callender, E.; J. Contam. Hydrol. 2007, 91, 4 .

6. Ellis, P. A.; Mackay, R.; Rivett, M. O.; J. Contam. Hydrol. 2007, $91,58$.

7. Mahler, B.; Massei, N.; J. Contam. Hydrol. 2007, 91, 81.

8. Felipe-Sotelo, M.; Andrade, J. M.; Carlosena, A.; Tauler, R.; Anal. Chim. Acta 2007, 583, 128.

9. Raiswell, R. W.; Brimblecambe, P.; Dent, D. L.; Liss, P. S.; Environmental Chemistry, Ediciones Omega: Barcelona, 1983.

10. Lu, X. Q.; Werner, I.; Young, T. M.; Environ. Int. 2005, 31, 593.

11. Drever. J. I.; The Geochemistry of Natural Waters: Surface and Groundwater environments, $3^{\text {rd }}$ ed., Prentice Hall: Englewood Cliffs, 1997.

12. Nguyen, H. L.; Leermakers, M.; Osán, J.; Török, S.; Baeyens, W.; Sci. Total Environ. 2005, 340, 213.

13. Gaur, V. K.; Gupta, S. K.; Pandey, S. D.; Gopal, K.; Misra, V.; Environ. Monit. Assess. 2005, 102, 419.

14. Tessier, A.; Campbell, P. G. C.; Bisson, M.; Anal. Chem. 1979, 51, 844.

15. van Oorschot, I. H. M.; Dekkers, M. J.; J. Int. 2001, 145, 740.

16. van Oorschot, I. H. M.; Dekkers, M. J.; Havlicek, P.; J. Int. 2002, 149, 106.

17. Barbeau, K. A.; Moffett, J. W.; Environ. Sci. Technol. 1998, 32, 2969.

18. Lã, O. R.; Barra, C. M.; do Amaral Sobrinho, N. M. B.; Mazur, N.; Velloso, A. C. X.; Quim. Nova 2003, 26, 323.

19. Oscarson, D. W.; Huang, P. M.; Liaw, W. K.; Clays Clay Miner. 1981, 29, 219.

20. Quan, S. X.; Bin, C.; Anal. Chem. 1993, 65, 802.

21. Piper, D. Z.; Wandless, G. A.; Environ. Sci. Technol. 1992, 26, 2409.

22. Torres, R. J.; Abessa, D. M. S.; Santos, F. C.; Maranho, L. A.; Davanso, M. B.; Nascimento, M. R. L.; Mozeto, A. A.; J Soils Sediments 2009, 9, 420 .

23. Janke, H.; Yamada, T. M.; Beraldo, D. A. S.; Botta, C. M. R.; Nascimento, M. R. L.; Mozeto, A. A.; Braz. J. Biol. 2011, 71, 903. 
24. Saraiva, V. K.; Nascimento, M. R. L.; Palmieri, H. E. L.; Jacomino, V. M. F.; Quim. Nova 2009, 32, 1995.

25. Silva, L. A.; Coelho, L. M.; Rosolen, V.; Coelho, N. M. M.; J. Braz. Chem. Soc. 2011, 22, 2094.

26. Bevilacqua, J. E.; Silva, I. S.; Lichtig J.; Masini, J. C.; Quim. Nova 2009, $32,26$.

27. Santos, M. L. P.; Santos, J. S.; Santos, J. R.; Oliveira, L. B.; Quim. Nova 2008, 31, 1997

28. Santos, J. S.; Santos, M. J. S.; Santos, M. L. P.; J. Braz. Chem. Soc. 2012, 4, 718.
29. Santos, J. S.; Santos, M. J. S.; Santos, M. L. P.; Quim. Nova 2009, 32, 1534.

30. Curtius, A. J.; Seibert, E. L.; Fiedler, H. D.; Ferreira, J. F.; Vieira, P. H. F.; Quim. Nova 2003, 26, 44.

31. CONAMA, Conselho Nacional do Meio Ambiente; Resolução 357/05; http://www.mma.gov.br, acessada em Julho 2012.

32. Oliveira, C. N.; Campos, V. P.; Medeiros, Y. D. P.; Quim. Nova 2010, 33, 1059.

33. Leroyer, A.; Hemon, D.; Nisse, C.; Bazerques, J.; Salomez, J. L.; Haguenoer, J. M.; Sci. Total Environ. 2001, 267, 87. 


\section{DISTRIBUIÇÃO DE Zn, Pb, Ni, Cu, Mn E Fe NAS FRAÇõES DO SEDIMENTO SUPERFICIAL DO RIO} CACHOEIRA NA REGIÃO SUL DA BAHIA, BRASIL

José Soares dos Santos*, Flávio Mendes de Souza e Maria Lúcia Pires dos Santos

Departamento de Ciências Naturais, Universidade Estadual do Sudoeste da Bahia, Estrada do Bem Querer, km 4, 45083-900 Vitória da Conquista - BA, Brasil

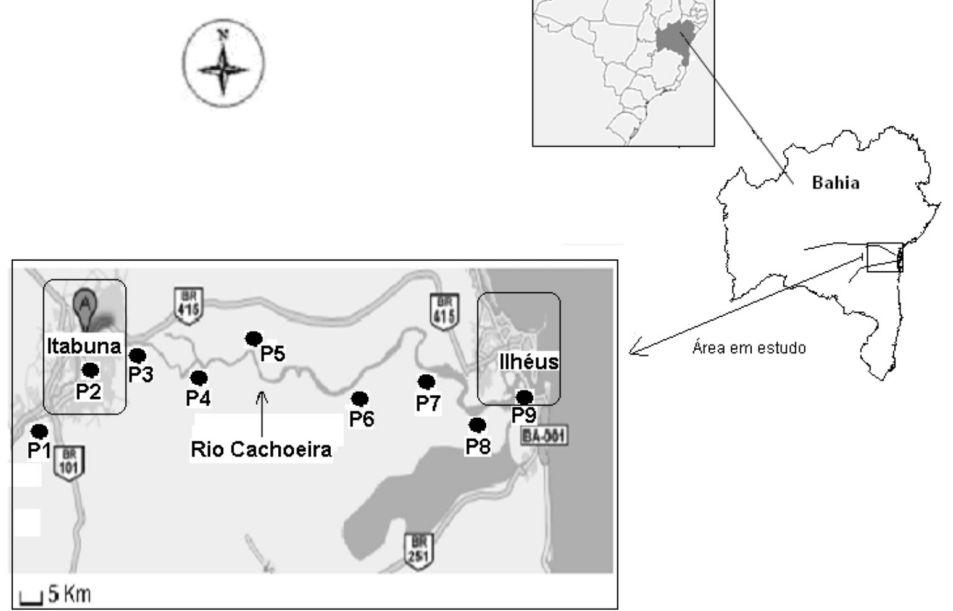

Figura 1S. Área em estudo e os respectivos pontos de coleta
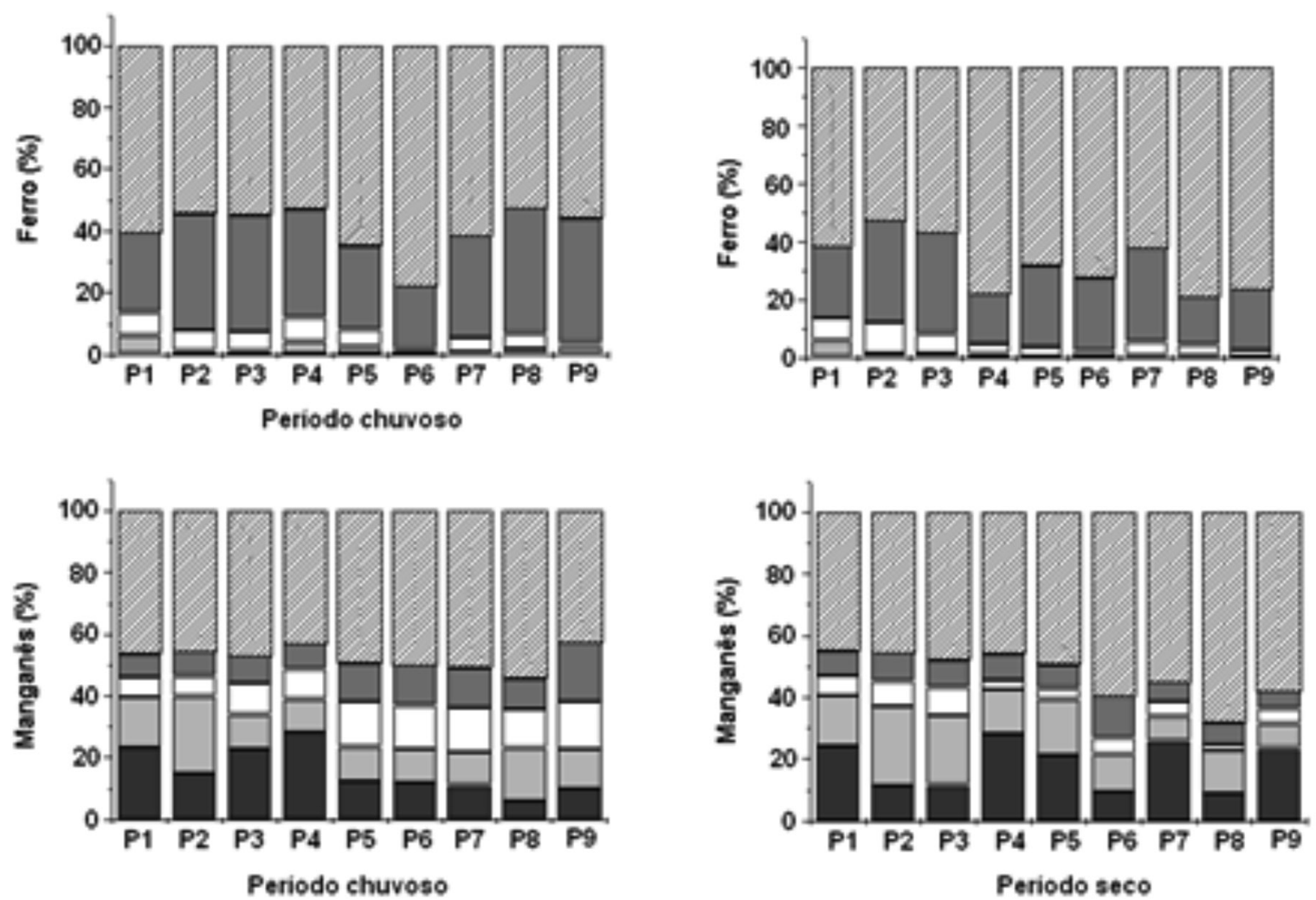

Figura 2S. Percentagens de ferro e manganês distribuídos nas frações do sedimento 

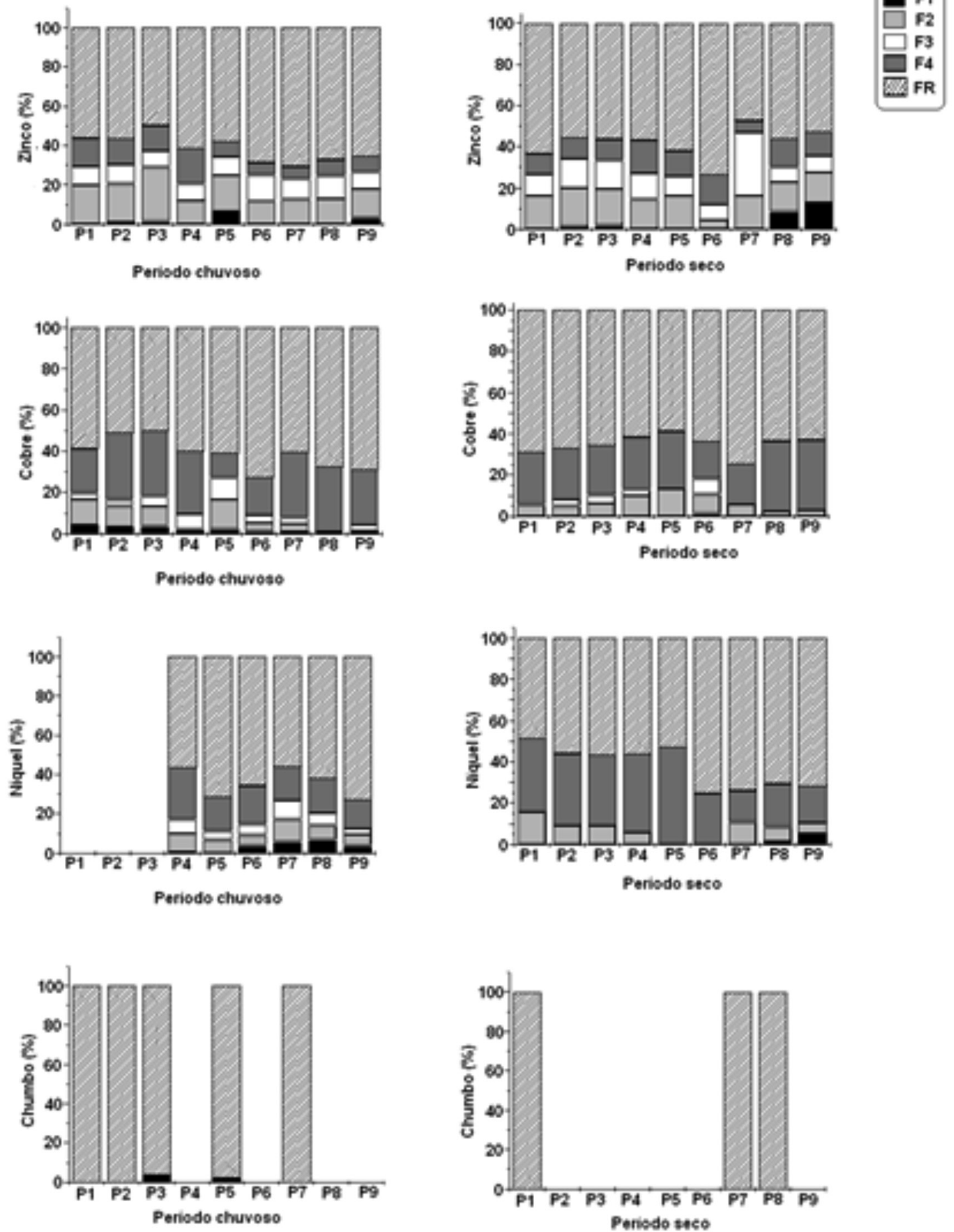

Figura 3S. Percentagens de zinco, cobre, níquel e chumbo distribuídos nas frações do sedimento 